\title{
Design and Implementation of the Management System of Large-scale Sports Event
}

\author{
Yuehua Li \\ The PE department of Baotou Teachers' College, Baotou 014030, China
}

Keywords: competition organization, theoretical analysis, management, large-scale sports event, public sports

\begin{abstract}
Under the globalization development background of the international sports event, along with our country's economic and social constant development, sports industry becomes the hot spot of the new sports developmental strategy which takes the sports event as the backing, looks the society and the economic development as the main purposes, and develops the new economic growth as the essential target .However, compared with vigorous development of sports event practices, the management level is obvious lag, especially the large-scale sports event's management is weak and difficult. So based on the above situation, the author chooses the organization as the research theme and tries to build the organization structure model of the different sports events in our country and analyses the essence and rules of the rational classification based on the sports' respective characteristics and the game theory between the government function and the market function framework. It is hold that the subject has important theoretical and practical meanings.
\end{abstract}

\section{Introduction}

According to systematic theory, any organization can be regarded as an integral and open system or a subsystem. Management is a process to achieve a goal by means of reasonably distributing resources, and the process also embodies the essential ideas of systematic optimization. With the enlightenment of systematic theory, this study is trying to find effective managerial means in reforming sports training and competing organizations systematically, thereby to optimize the allocation of personnel, finances and materials etc. in these organizations [1].

The Organization and management of Large-scale Sport Events is of Large-scale Sport Events organization and management in a variety of activities and effectively do the work of the organization of Large-scale Sport Events, categorized, with the authorized personnel and the division. The long years of practice organization and management of large-scale sporting events is an extremely complex social system project [2]. Therefore, based on the system of scientific thought and modern systems engineering methodology $[3,4]$, to rely on modern science and technology to carry out the organization and management of Large-scale Sport Events is an inevitable choice. Large-scale Sport Events organization and management information system is an integrated use of modern information networks and modern digital technology, by streamlining the internal and external management of its Large-scale Sport Events organization and services, optimization, integration and restructuring to achieve the efficient operation of information systems tools. By using this system to break the constraints of time, space and departmental separation, integration of efficient, high-quality, honest communication of information services for the successful hosting of Large-scale Sport Events.

When discussing the "length and breadth" of sport training system in China, we found that the main conflict, which lies in the process of organizing and actualizing the "sports system", "education system" and "social system", is the problem of "governing system". Presently it is concerned with how to solve the problems like "reasonably utilizing personnel, finances and materials", "learning and training" and "physique and mentality". Therefore, the thesis suggests to the sport training system in China that, in the near future, by the macro-leadership of sport management center of General Administration of Sport, we should reasonably and systematically set up and organize, the arrangements, departments, responsibility and right of the sport training; in the long term, we should 
break the status quo that while managing sport affairs, the three systems, "sports", "education" and "society", have confused the systems, responsibility and right. We should build a sport administrative network based on the association system.

When discussing the "length and breadth" of sport competing system in China, we find that "sports and education system" has not built a perfect-running mechanism when it manages and holds competitions. Therefore the thesis suggests to the sport competing system in China that, in the near future, by the macro-leadership of sport management center of General Administration of Sport, we should systematically and reasonably arrange various competitions of all levels; in the long term, we should take middle and primary school sport matches as the pyramid base, college matches as the body, professional league matches as the peak, then we can form a sport competing system, which has clear administrative levels, definite responsibility and right, reasonable rules, perfect match arrangements, and operating orderly.

At present, our country's large-scale sports event management level is not high so far, holding sports event is too depending upon the resources superiority which brings by whole nation system, the sports event benefit brought by management is still insufficient, the fundamental research is weak. In large-scale sports event management field, the fundamental research achievement relatively is still weak, did not pay enough attention to our country specific background system, lacks integration research and the empirical study. This obtained the expert and scholar's confirmation in the process of investigation. Based on the research on materials combing with the inspection of the whole process of large-scale sports event organization and operational guidance, proposes the separation of "the organization pattern" and "the operational guidance pattern" of the large-scale sports event, includes "the sports event resources deployment" and "the sports event resources using" two sports event work category which contents and the nature are different.

\section{The Basic Statue of Large-scale Sports Event and Management Organization}

The market operation and management mode of government participation is the inevitable requirement of the Olympic Games held successfully. This combination-type organization and management models combine's government with market can complement each other, complementary advantages, so as to realize the Olympic Games in the political, economic, culture, educational and social value. The policy and system has determined that the Government has functioned as the event provide, has become the main means of resource allocation in events, however With the perfection of socialism market economic system, the funding of major sports events will be progressively market-oriented, diversified, which will replace the Government to become the primary means of resource allocation. "Venulization" operation and management mode, transfers from the type of competition organization structure to the matrix, and realizes the dual goals of function specialization and competition characteristics. This pattern strengthens horizontal linkages, combining centralization and decentralization; strengthens organization's mobility, flexibility and coordination; released professionals' potential abilities and avoided the waste of resources for project board mode. The setting and location of China's major sports events organizational structure geared to international standards is the necessary requirement. Refinement of tasks makes the organizations constantly adjust and improve, contents constantly expanding. The establishment of committee organizational structure intensified the tasks and goals, and ensured the realization of the final goal.

National games operated through the company's operations, and used the market-oriented resources allocation, fully mobilized all our efforts and attracted a large number of manpower and material resources; Outsourcing-type organizational structure model is the functional specialization and goal-oriented product. However, compared with vigorous development of sports event practices, the management level has obvious lag. Especially the large-scale sports event's management is the weakness and the difficulty. We take "Organizing pattern innovation and the optimizing operational practice of large-scale sports event of our country" as the main study object, study and refines the optimizing elementary management theory of large-scale sports event in our country, with the help of sociological social environment theory, western economic public choice theory, process management 
of management principle theory and so on.

\section{Establishing and Perfecting the Crisis Management System}

During the 100 years when the modern sport developed, sport events, home and abroad, experienced lots of crises, and people learnt some lessons and accumulated some experiences from them. Some of the crisis event still not far from us and we should take warning. From these crisis events, we can know that the crises which are coursed by natural disaster takes a little part, but most by anthropogenic factor, the crises which are coursed by political resistance and war also fade out, and the crises that are coursed by environment problems, public sanitation and terrorist attack become the problems. The crisis in sport events can easily become the social crisis, because the sport events are usually held in the cities where are populous, and have high movement of population and transportation and information relatively developed. And there are close relationships among people and developed media transmitting system which can quickly spread the crisis, if the responses are improper, it could be a regional crisis or even a national crisis. Through the occurrence, development and response of the crisis in sport events, we can know that every crisis contains the roots for failure and the seeds for success. We should have a prompt reaction to the crisis and reflect on the whole process of the crisis, and we can take some lessons and accumulate some experience, so that to raise the speed of reaction and avoid the crisis next time, and minimum the damage which is coursed by crisis. Meanwhile, we should realize that no matter how well you prepare for the following crisis, there are always oversight and insufficiency. The perfect preparation is the perpetual summary of the lessons, the discovery of shortcomings, the ability of reactions and maximum decreasing of the damage which is coursed by crisis.

The establishment of the crisis management system is the guarantee of the realization of the crisis management. It bases on the regular science and democracy and the irregular high efficiency and sensitivity. In the strong crisis management system, it should contain four major systems that is the authoritative conduction organization system, the systemic organization system; the powerful integrate coordination system and the high quality of information and advisory organization system, as well as the ordered, reasonable and coordinative organic system. Crisis management system should adopt different strategies according to the three periods: latent period, outbreak period and recovery period. It includes the pre-alarming, response and recovery of the crisis.

The crisis pre-alarming system is a sensitive and accurate information detecting system. It can collect the relative information promptly, and then analyze and deal with it. It can also take auto diagnosis regularly or irregularly and evaluate the condition of the running of the organization objectively, in order to catch the omen of organization crisis and take firm measures. The handlings of the crisis not only prevent the further expanding of the crisis and limit the damage from the range and degree, but resolve the crisis effectively. And the essential part is the "optimistic measurements" which bases on the "pessimistic preparation". After the overcome of the crisis, one important task is to make and adjust the plan and carries out daily work again, to make the organizing committee and working order restore the regular condition, to compensate the dead and wounded during the crisis and restores the facilities and buildings. And also it should summary learns from the experience and make relevant modification, in order to enhance the immunologic function of the organizing committee, and prevent and avoid the crisis. Though the natural disaster can't avoid positively, through the effective measurement, the effect and the degree of damage can be alleviated.

During the handling of the crisis, one important element is the prompt and accurate communication of the information, especially the leading of the public for the crisis issues. After the crisis, every situation is indefinite, and because of the dissymmetry and imperfection of the information, the public can easily produce scared mentality. The rapid publication of the crisis and different protecting measurements not only improve the precaution consciousness and know the relative precaution knowledge, but appease the public mood, avoid the panic-stricken, stop various rumors and hearsays and prevent the social unsteadiness which are coursed by understand deviation of the crisis. Sport events, especially Beijing 2008 Olympic Games, will gain great attention all over the world, and every media, every country and region will report this big game. The intervention of 
television, newspaper, radio and Internet will be all-around and deep, that is to say, everything in the Olympic is not a trifle, and it can't flee from the reporters' eyes. Therefore, as long as the crisis appears in Olympic Games, the prompt, objective, accurate and complete information should be reported formally, officially and systematically. And it should make the public feel the organizing committee's sincerity and confidence, so as to extinguish the negative effect of the crisis among the athletes, audiences and the citizens of the host city.

The smooth holding of sport events not only needs the endeavor of the native people, but the cooperation among nations, especially the handling of the outburst public safety event. The international and integrative sport events, like the Olympic Games, tend to be utilized by some terror makers. The two crisis events in Olympic history show that the terrorists can be home or abroad, and their aims may be at the host country or the other countries in the running. The reasons for the terrorism are complicated (racial contradiction, society dissatisfaction, etc. and various, and they distribute widely and hard to prevent. Meanwhile, the appearance of the suicide attack added the challenge for countertenor. The cooperation among nations can help the organizer learn the successful example and the latest theory patterns of the international large-scale activity directly, and it also can help the organizer make the plan and take the measurements for the sport events, in order to ensure the smoothness of the sport event.

The process of sport event operation is consisted of planning, organizing, implementing and evaluating. Sport event is the area that the various crises frequently happen. The crisis management in sport court has arisen much attention. Whenever the sport event is held, the safety protecting should be reinforced. Because of the lack of effective management system, the crisis still happened in sport events. Therefore there is magnificent meaning to research the crisis management for sport events, avoid the transfer of the unexpected event to the crisis and the maximum guarantee of the potential risk and damage and the minimum lose of the manpower, financial resources and environment which are coursed by outburst crisis.

\section{Conclusions}

Our country has held Beijing 2008 Olympic Games and Harbin 2009 World University Games and other sport events. There may be some risks and crises during these sport events, but if we want to make the maximum function on the economy, culture and politics of the host country through these sport events, some effective measurements must be taken and the organization and manage work of large scale sports event should be carefully considered based on scientific decision. We should also notice the weak points and improve the handling ability, and try our best to minimum the negative effect and damage of the crisis and also the conflicts brought by bad organization, and also should make sure the smoothness of the sport events.

\section{References}

[1] Jie Dong, Xinli Liu and Luyi Song, Risk Management of Emergency for Beijing 2008 Olympic Games Sports \& Science, 1:30-35, 2012

[2] Ping Ling , Jie Tong. Risk Management on Beijing 2008 Olympic Games Zhejing Sport Science, 12: 4-6, 2013

[3] Lei Fang, Wenqing Zhang. Research on Framework of Government's Crisis Management System under the Circumstance of E-Governance China Soft Science, 4: 32-36, 2011

[4] Yan Qin. A Research on the Crisis Management for Important Athletic Events Journal of Capital Institute of Physical Education, 4: 25-28, 2012 